\title{
The Application of Database Technology in the Network Management System
}

Yong-qiang HE, Xue-rui WANG

Henan Institute of Engineering

Zhengzhou - 450007

China

\begin{abstract}
In today's society, the internet was increasingly expanding the scale of the computer network, the structure of the network has become more and more complex, the network management system must have a higher intelligence. A large amount of data had been processed in the network management system, and the application rules on data processing are the two most basic elements, and the database had provided a technical solution to achieve the function of these basic elements. This paper discussed the network management system, the basic function of network protocols and network management MIB databases, the ERC plus method of database design are discussed, and the application example is given. The article also analyzed the adaptability of network management application on database. The design and maintenance of the network system is very useful.
\end{abstract}

Keywords: Database, Network Management System, Network Management, Protocol Management Information Base, ERC Plus Method

Received: 14 March 2019, Revised 27 June 2019, Accepted 2 July 2019

DOI: $10.6025 /$ jnt $/ 2019 / 10 / 3 / 87-91$

(C) 2019 DLINE. All Rights Reserved

\section{Introduction}

As a new type database technology, data management of object oriented database system technology has begun to be used deeply in the transaction work areas of enterprise. According to the present application situation of database, data management of object oriented database is more scientific and timely, according to the extension and development of database technology, this paper introduced and analyzed the characteristics of object oriented database and how to establish object oriented database, the author of this paper thought that the data planning, the overall data analysis and the data design should be focused on during the establishment of whole system. A perfect network management system was the guarantee of the computer network reliable and stable operation, also the basis of analysis of network performance. And the network management system used database to store information about network and its operation, of which the database was called MIB, Management Information Base. The implementation of MIB, to a large extent, depended on the object oriented design thought. Each network monitored and controlled resources was marked by the management object, so the MIB became a structured collection of these objects. It is worth to pay attention to these collections MIB, despite of using object-oriented design concept, it was not to say that the object-oriented database management system or object-oriented technology was the must way to implement it. As long as the transfer of information between open system was with the object-oriented design principles. So what kind of database could implemented MIB had became a question worth to discuss. can be discussed. Among the various kinds of database technology, the database depending on its own advantage realized MIB to open the door for connivance. 
With the development of computer application and the development of multimedia technology, the large information systems were based on design theory of relational database system. But these database system included hierarchical database, network database and relational database, and no matter what's the difference between the model and technique, but it mainly were for data management and support transaction processing applications. However, with the improvement of the user application requirements, the development of hardware technology and Internet/Intranet to provide colorful multimedia communication, promote the database technology and object-oriented programming technology, network communication technology mutual penetration, in combination with each other. It became the main characteristic of the current database technology development to absorb and make use of these new technologies and continuous efforts to meet the enterprise's information management needs.

\section{Database Technology Application Situation}

The application of database technology, whether it be a production information system, supply and marketing information system, or information systems asset management information system had been established. Data security, reliability was guaranteed, but the scientific nature data, data processing, feedback and penetration still needed further perfect the combination of science and technology.

\subsection{The Characteristics of the Object-oriented Database Technology}

Object-oriented database technology was objective to the world, a stable objective existence entity object as the basic elements, and to "class" and "inheritance" to express things have in common and internal relations between them. The related concepts of object-oriented and programming technology, the combination of object-oriented database system could take automatically retrieve the data as object storage and sharing, including the object to complete each database transaction processing instructions, these objects may contain different types of data, including the traditional data and processing, sound, graphics and video signal, object could be shared and reused. Object-oriented database system supported network communication applications and multimedia applications. These applications could combine different types of data, is very practical and convenient.

\subsection{How to Build an Object-oriented Database System}

Combined with the status quo analysis of object-oriented database system programming technology application in the actual work. We referred that the production management system, supply and marketing management, asset management system all needed to be a more perfect and convenient operating system, can store data. It can chad reads the data we need, but also to the data, we now created a simple database asset management system (reference source program 1 asset management database system class 1,2,3). The system there were two databases needed to collect, at the same time, one was equipment database, the other was a house or a database, so it needed to use an object-oriented database class to achieve the purpose of this database application management procedure. It can put the same data with associated attributed "class" together, the equipment was a kind of object, housing was another kind of object, they are the same interface on the space of two independent individuals, they have their own member variables. That device class could be constructed in the class variables being different, they have the function of public, the public function also had its own member functions, class object using the operator to access define respective member function, does not interfere with each other, Defined after their respective functions, they run in defines the scope of their respective, don't have to call them scope, and these categories are objects of data can be reused to regain, and automatic is this package and class inheritance in object-oriented programming are very common example. It used in an interface, object-oriented different types of different data, not used in a number of interface operation, saved a space resources is also reduced tedious programming provides a great convenience.

1. The source program 1 asset management database system class 1

4. using namespace

5. class machine

6. \{

7. private:

8. char cName[50];

9. char ctype[50]; 
10. float fjz;

11. public:

12. void Accept();

13. void Display();

14. using namespace zcgl;

16. class building

17. \{

18. private:

19. char cName[50];

20.int imj;

21. float fjz;

22. public:

23. void Accept();

24. void Display()

25. $\}$;

It can be seen clearly above procedure is for the entire database objects do statement, two classes were statement object, is a machine (equipment), the other one is building (housing), and they have a public member function the Accept () and Display (). The definition of their respective member function for the next step is prepared and bedding, they also have their own member variables, that is to say, on the basis of the same to allow the different of each type of data exists.

The source program 1 asset management database system class 2

26. int main()

27. \{

28. machine $\operatorname{macObj}=$ new machine():

29. building buiOhj=new building();

31. $\operatorname{macObj}$.Accept();

32. buiObj.Accept();

34. macObj.Display();

35. buiObj.Display();

36. return 0;

37. $\}$

The above program 2 began to define their respective class object instance, one was macObj (device class), the other was a buiObj (housing), and their respective member function had been defined, the Accept () function was a member function of a macObj. The Accept (). The other one was buiObj. The Display (), they were all defined as integer variables. Class object applications operator “. "to access the respective member function.

The source program 1 asset management database system class 3

39. void maRhine::Accept() 
40. \{

41. Cout $<<$ "Please enter the name of the device"

42. Cin >>cName;_—__ «ENDL; cout «?Equipment net value

43. coul $<<$ "Please enter the name of the device:"; Yes:? $<<$ fjz $<<$ endl; cout $<<$ ?Device Number

44. Cin $>>$ ctype; Yes:?<<ctype $<<$ endl; coul $<<$ ? nDevice Name

45. Cout $<<$ "Please insert device net value:"Yes:?<<cName $<<$ endl; $>>$ cName;

46. $\mathrm{Cin}>>\mathrm{fjz}$;

47. $\}$

48. void machine::Display()

49\{

50. Cout $<<$ " $\backslash$ n $—$ Show the device information

51.)

Above procedure 3 analyzed the application of domain decomposition operator "::" the member functions of the public functions were defined, the data in object-oriented database system were not only run in defining its scope. And these data object could also reuse and regain automatically, object-oriented database system was a kind of convenient operation, with a handle to the new data query, database technology.

\section{Other Database System Design Technology}

With the aid of network communication technology, distributed database design technology, distributed database management system (see figure 1) was the combination of distributed technology and database technology of database technology. Conceptually, a distributed database was physically dispersed in each node on the computer network, and logically it belonged to the same system of data collection. Distributed data system had the characteristics of: (1) most of the processing was immediately completed; (2) the computer was connected by the associated data communication network; (3), overcame the weakness with a central database, reduced the cost data transmission; (4), improved the reliability of the system, reduced local system fails, the other part still could continue to work; (5), the position of each database was transparent, convenient system expansion; (6), in order to coordinate the transaction activities of the whole system, the performance of the transaction management expense was still higher.

Distributed was the development direction of computer application in enterprise information, also the actual needs of the database technology application, its application areas: (1) A distributed database system was set up between the departments, and adapt to the distribution of the department organization structure, reduced the communication cost, improved the response speed, and made these departments use database more convenient and economy; (2) When there was something wrong between, a place did not cause the whole system collapse, because failure site users could log in the system through other sites, and other site users could also access path selected by the system automatically to avoid the fault sites, using other data copy operation did not affect the normal operation of the business, so as to improve the reliability and availability of system;(3) when in a large enterprise or department had completed a number of the database, in order to exploit the resources of each other, and develop the global application and develop a distributed database system, it needed to make full use of the database resources; (4) when a department or the field scale need to expand or increase, the structure of distributed database system for the processing capacity of extended system provided a good way: Added a new node in distributed database system to do so than in a centralized system expand the scale of the system was much more convenient, flexible, and economic, so as to achieve a gradual extension processing capacity and the size of the target system. However, the structure of the distributed system, distributed database and its implementation technology, the problem of the current did not achieve the desired goal. Because the information management platform was in the further perfect the process of construction, sharing and network collaborative work had been on the agenda, overcame its implementation technology and the difficulty of hardware and software environment, was the enterprise information management system of modern management of the trend of the times.

Journal of Networking Technology Volume 10 Number 3 September 2019 


\section{Conclusion}

Our vast database of users were with the hope to get what they need data or information, and the ability to easily accept and use these data or information. The post requirements shall be through hardware and software engineering environment support and high quality database design to achieve; the Last kind of requirement the user improve good user interface and application support to achieve perfect. But it was important to note that any database system, the fundamental problem was the recognition of application in the field of basic data and organization, if can't do this, it was hard to make customer satisfaction and recognition of database system. As a result, the database system construction planning, comprehensive database for application in the field of comprehensive data analysis and design, would be a top priority in the construction of the system.

\section{References}

[1] Ji-min SHI., Guan-quan TANG. (2004). Database application system development tutorial, Beijing: Tsinghua University Press.

[2] Bo-le SHI., Ning GU., et al.(2001). Translated. [USA] Devid M Kroenke Database processing. Beijing: electronic industry press.

[3] Wine miller[M], E. (2001). Visual Basic 6.0 Database development. Beijing: tsinghua university press.

[4] Data, C J. (2013). An introduction to database system. Beijing: mechanical industry publishing house.

[5] ITU X 781-2001 Guidelines for defining CORBA managed objects.

[6] Lupu, E. C., Sloman, M. (1999). Conflicts in policy-based distributed systems managements. IEEE Transactions on Software Engineering,1999 (06) 850-858. 